\section{Reducing mortality in diastolic heart failure: a role for statins?}

There are no proven therapies for improving survival in patients with diastolic heart failure, which is defined as heart failure with a normal ejection fraction. Drugs known to lower mortality in heart failure patients with a reduced ejection fraction might, however, be useful in this setting. Preliminary work by Fukuta and colleagues has now shown that diastolic heart failure patients might benefit from the use of statins.

A total of 137 consecutive patients with heart failure and an ejection fraction of 0.50 or above were followed up for a mean period of $21 \pm 12$ months. At the point of study entry, patients were treated with angiotensinconverting-enzyme inhibitors or angiotensinreceptor blockers (55\%), $\beta$-blockers $(50 \%)$ or calcium-channel blockers (27\%). None of these therapies was associated with improved survival. The 68 patients (50\%) treated with atorvastatin, simvastatin, pravastatin or fluvastatin, however, were shown to be at a significantly lower risk of death than those not receiving statin therapy. After adjusting for variables such as hypertension and coronary artery disease, the relative risk of death in the statin group was 0.20 (95\% Cl 0.06-0.62).

Fukuta et al. discuss possible mechanisms by which statin therapy might reduce mortality in diastolic heart failure patients-including a reduction in left-ventricular hypertrophy and fibrosis and an increase in arterial distensibility-and call for a randomized study in this area.

Ruth Kirby

Original article Fukuta $\mathrm{H}$ et al. (2005) Statin therapy may be associated with lower mortality in patients with diastolic heart failure: a preliminary report. Circulation 112: 357-363

\section{4-slice CT: a comparison with invasive coronary angiography}

The latest 64-slice CT scanners promise to provide highly accurate, noninvasive measurement of coronary artery stenoses. Leschka and colleagues have recently presented the results of their initial experience using this new technology.
This prospective study included 67 patients who underwent invasive coronary angiography for suspected coronary artery disease. All arteries of more than $1.5 \mathrm{~mm}$ diameter were examined, and a reduction in diameter of greater than $50 \%$ was considered significant. Within a median interval of approximately 2 weeks, all patients were also investigated using 64-slice $\mathrm{CT}$, and the results from the two techniques were compared.

Invasive angiography identified 20 patients without significant coronary stenoses, all of whom were also correctly identified by 64 -slice $\mathrm{CT}$. The remaining 47 patients had significant stenoses on angiography. These lesions were detected by CT with a sensitivity of $94 \%$ and a specificity of $97 \%$. The corresponding positive and negative predictive values were $87 \%$ and $99 \%$, respectively.

These initial results suggest that 64-slice CT is a highly accurate means of assessing patients with coronary artery disease, which might represent a useful, noninvasive alternative to invasive coronary angiography. The researchers discuss the limitations of the new technology and note that further studies will be needed to determine appropriate threshold values for vessel size and heart rate.

Ruth Kirby

Original article Leschka S et al. (2005) Accuracy of MSCT coronary angiography with 64-slice technology: first experience. Eur Heart J 26: 1482-1487

\section{'Normal' urinary albumin excretion and cardiovascular risk}

In patients with hypertension or diabetes, microalbuminuria-a urine albumin to urine creatinine ratio (UACR) of between 30 and $300 \mu \mathrm{g} / \mathrm{mg}$-is a known risk factor for cardiovascular disease. A community-based study has now revealed that urinary albumin excretion below this range (UACR $<30 \mu \mathrm{g} / \mathrm{mg}$ ) might also be associated with an elevated cardiovascular risk.

A group of 1,568 Framingham Offspring Study participants without hypertension or diabetes were followed up for a median period of 6 years, during which 54 incident cardiovascular events and 49 deaths were observed. The median UACR values (assessed on a spot 